\title{
The Contribution of Galenics to Patients' Sensory Perception of Nasal Sprays After Nasal Surgery: Data from a Prospective Randomised, Controlled, Double- Blind, Crossover, Multicentre Study
}

\author{
Christoph Bergmann · Jennifer Lander · Lea Radtke · Laura England • \\ Jaswinder Singh · Ralph Mösges (D)
}

Received: August 26, 2021 / Accepted: September 27, 2021 / Published online: October 24, 2021

(c) The Author(s) 2021

\begin{abstract}
Introduction: Postoperative care after nasal surgery is commonly achieved with nasal sprays. The current study compared two decongesting, wound-healing nasal sprays in patients after nasal surgery in order to investigate their sensory perception. One of the sprays
\end{abstract}

Christoph Bergmann and Jennifer Lander contributed equally to the manuscript.

Supplementary Information The online version contains supplementary material available at https:// doi.org/10.1007/s12325-021-01937-X.

C. Bergmann

Medical Faculty, University of Essen, 45147 Essen, Germany

C. Bergmann

RKM 740 Interdisziplinäre Facharztklinik, Pariser

Str. 89, 40549 Düsseldorf, Germany

J. Lander

Faculty of Medicine, RWTH Aachen University, Pauwelsstraße 30, 52074 Aachen, Germany

L. Radtke $\cdot$ L. England · J. Singh · R. Mösges $(\bowtie)$ ClinCompetence Cologne $\mathrm{GmbH}$, Theodor-HeussRing 14, 50668 Cologne, Germany

e-mail: ralph@moesges.de

\section{R. Mösges}

Institute of Medical Statistics and Computational Biology (IMSB), Faculty of Medicine, University of Cologne, Kerpener Str. 62, 50937 Cologne, Germany was a new galenic formulation (nasic ${ }^{\circledR}$ neo, Cassella-med GmbH \& Co. KG).

Methods: According to the crossover design, patients who had undergone nasal surgery applied two different nasal sprays during two treatment periods of 4 days each, interrupted by a 3-day washout period. Sensory perception of the nasal sprays was assessed with the nasal spray sensoric scale. Throughout the study, nasal obstruction was evaluated by patients, and physical examinations, measurements of vital parameters and rhinoscopic examinations were carried out by investigators. Adverse events were documented during the entire study, and following treatment, patients judged the overall preference, efficacy and tolerability of both products.

Results: Overall, no significant differences in sum scores of the assessments of the nasal spray sensoric scale were observed between treatments. A significant period effect observed during the crossover study limited the overall analysis. Nevertheless, significantly more patients preferred the new galenics nasal spray compared to the comparator spray $(57.1 \%$ vs. $34.7 \% ; p=0.031)$. Further, $10 \%$ more patients rated the efficacy of the new galenics as 'good' to 'very good' compared to the comparator. Importantly, a subgroup population of patients with more pronounced signs of inflammation present at screening evaluated the sensory perception of the new galenics as significantly better $(p=0.033)$ compared to the comparator. 
Within this subgroup, no period effect was observed. The application of both nasal sprays was shown to be safe and well-tolerated.

Conclusion: The overall sensory perception of both nasal sprays was evaluated comparably well in patients after nasal surgery and overall the application of the new galenics nasal spray was preferred by significantly more patients compared to the comparator nasal spray. Patients with marked nasal abnormalities may have a greater benefit from the contribution of galenics as significant differences in the sensory evaluation by the nasal spray sensoric scale in favour of the new galenics product were shown for this subgroup.

Trial Registration: The current study was registered in the EU Clinical Trials Register with the EudraCT No. 2019-004936-52.

Keywords: Nasal Spray Sensoric Scale; Nasal Surgery; Rhinopathia; Sensory Perception; Treatment Satisfaction

\section{Key Summary Points}

Nasal symptoms occurring after nasal surgery or during acute rhinitis are commonly treated with decongesting, wound-healing nasal sprays. As the galenics of nasal sprays influence their sensory perception, changes in galenics may have a great impact on patient compliance

The study compared the sensory perception of two decongesting, dexpanthenol-containing nasal sprays with (new galenics) or without (comparator) hyaluronic acid, hypothesizing that the changed galenics may improve the sensory properties of the new galenics product

Although no significant differences regarding the sensory perception of the nasal sprays were observed between treatments because of a strong period effect, a greater proportion of patients $(p=0.037)$ preferred the new galenics nasal spray over the comparator spray
A subgroup of patients manifesting more pronounced inflammation signs at screening assessed the sensory perception of the new galenics nasal spray better $(p=0.033)$ compared to the comparator spray, indicating that the changed galenics might be beneficial for those patients

\section{INTRODUCTION}

The main functions of the nose are respiration with heating and humidification of the inhaled air (air conditioning), particle filtration via the mucociliary transport system, and the olfactory function [1]. These functions are influenced by the variable cavernous tissue of the nasal septum and the nasal conchae, as the mucosa covering both structures regulates the width of the nose and its functional state.

In clinical practice, several reasons, e.g. dysmorphoses, nasal obstruction or mucus hyperproduction, may result in functional impairments of the nose, and corrections of the nasal septum and the nasal conchae, especially the lower concha, are among the most frequent surgical interventions in otorhinolaryngology [2].

Every surgical intervention represents an injury to the tissue, to which the body reacts with a constant inflammatory reaction. The aim of this is to fend off pathogenic germs, to break down necrotic tissue and to restore the tissue structurally and functionally with the help of proliferation and repair processes. After an operation on the nasal septum or the nasal conchae, postoperative symptoms such as a blocked nose, runny nose, or reduced sense of smell often occur in addition to pain and bleeding. For symptomatic treatment in the postoperative period, various active ingredients or combinations of active ingredients are recommended, including the use of saline solutions for humidification, steam inhalations, decongestants and intranasal steroid preparations. 
According to the currently valid guidelines, isotonic nasal sprays or appropriate nasal rinses are used after nasal surgery to soothe the nasal mucosa, and decongestant nasal sprays and ointments can alleviate the typical symptoms of dry crust and bark formation [3]. The combination of the decongestant xylometazoline hydrochloride and the wound-healing dexpanthenol has been established as standard postoperative nasal wound care over the last 20 years $[4,5]$. Decongesting nasal sprays are also recommended for treatment of acute rhinitis [6]. As symptoms in patients after nasal surgery and with acute rhinitis have common features (e.g. presence of nasal congestion and irritations of the nasal mucosa), treatment after nasal surgery represents a good model for acute rhinitis and the results of the current study are of relevance for both indications.

The current study aimed to compare the sensory quality of two nasal sprays containing the combination of xylometazoline hydrochloride and dexpanthenol, one of which (nasic ${ }^{\circledR}$ neo) additionally contained the excipient hyaluronic acid resulting in a new galenic formulation (for detailed formulations, see "Methods"). On the basis of the assumption that these changed galenics have a positive impact on the sensory properties, the perception was studied in patients following nasal surgery.

\section{METHODS}

\section{Study Design and Ethics Compliance}

The current study was a prospective, doubleblind, randomised, controlled, multicentre, crossover trial, which was carried out in three ear, nose and throat outpatient centres in Germany.

The study was conducted in accordance with the principles of the current (2013) version of the Declaration of Helsinki, Good Clinical Practice (CPMP/ICH/135/1995), the study protocol, requirements of the German Medicines Act (AMG), the Basic Data Protection Regulation (DSGVO) and other applicable regulatory requirements.
Approval of the current study was granted by the Ethics Committee of the Ärztekammer Nordrhein (medical council North Rhine, reference number 2020061). The approval covered all participating study centres as all three study centres were located within the district of the responsible ethic committee. Therefore, in accordance with the AMG, the favourable vote of this responsible ethic committee was valid for all three study centres.

All patients provided informed consent prior to participating in this study. No data identifying single patients were included in this study; therefore, informed consent for publication is not needed.

\section{Study Patients and Treatments}

Female or male patients (18-64 years) with postoperative nasal breathing disorders after surgery on the nasal septum or the nasal conchae were eligible to participate in the trial. Details of inclusion and exclusion criteria as well as allowed and forbidden medications are listed in Table S1 in the supplementary material. After signing an informed consent, patients were randomly allocated to treatment sequence 1 (new galenics, followed by comparator treatment) or 2 (comparator treatment, followed by new galenics; Fig. 1). Randomisation was ensured by block randomisation with a block length of 6 , and randomisation lists were transferred to an external pharmacy for packaging of the investigational products. Patients were assigned ascending treatment numbers in chronological order of appearance at the trial site, and investigational products were applied by patients according to the assigned treatment sequence.

Treatment was applied in crossover design: the respective first nasal spray was applied for 4 days (days $0-3$ /treatment period 1 ), followed by a washout period of 3 days (days 4-6), continued by application of the second nasal spray for another 4 days (days 7-10/treatment period 2, Fig. 1). During the treatment periods, patients applied the nasal sprays upon demand but a maximum of one spray per nostril three 


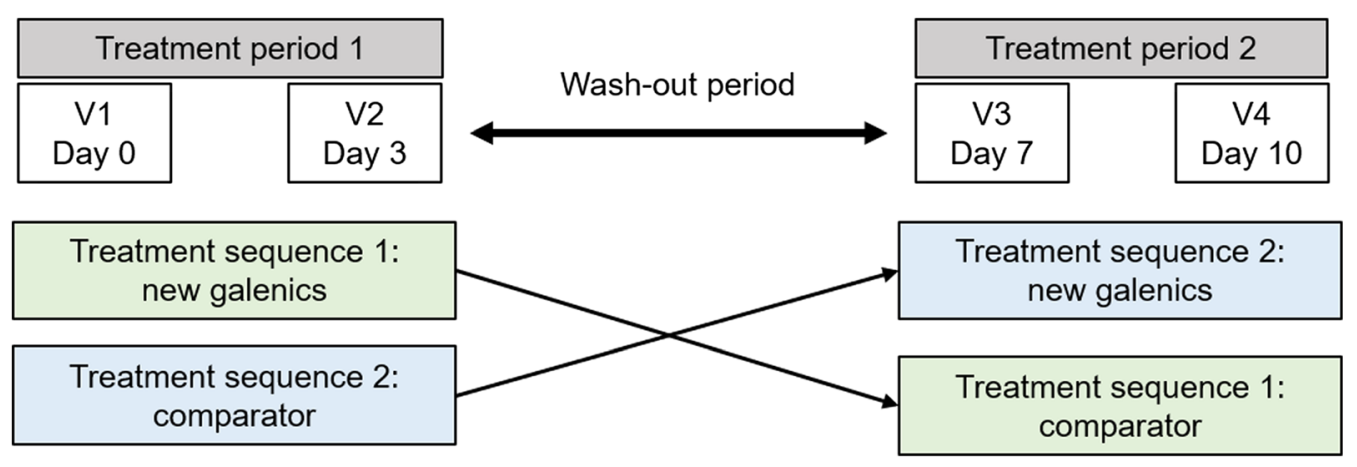

Fig. 1 Crossover design and visit time points of the trial. During the first visit (V1) on day 0, patients were randomly allocated to treatment sequences 1 or 2 . Patients of treatment sequence 1 applied the new galenics product for the first 4 days $(\mathrm{d} 0-\mathrm{d} 3)$ followed by a washout period

times a day; during the washout period, patients did not use any of the investigational products.

The investigational medicinal product (IMP) used in the trial was the nasal spray nasic ${ }^{\circledR}$ neo (hereafter referred to as new galenics) (containing the active ingredients xylometazoline hydrochloride $[1 \mathrm{mg} / \mathrm{mL}]$ and dexpanthenol [50 $\mathrm{mg} / \mathrm{mL}]$ and the excipients potassium dihydrogen phosphate, sodium monohydrogen phosphate dodecahydrate, sodium hyaluronate and purified water; Klosterfrau Berlin $\mathrm{GmbH}$ [manufacturer], Cassella-med $\mathrm{GmbH} \& \mathrm{Co} . \mathrm{KG}$ [marketing authorization holder]). The comparator product was the nasal spray NasenDuo ${ }^{\circledR}$ (containing the active ingredients xylometazoline hydrochloride $[1 \mathrm{mg} / \mathrm{mL}]$ and dexpanthenol $[50 \mathrm{mg} / \mathrm{mL}]$ and the excipients potassium dihydrogen phosphate, disodium hydrogen phosphate and water for injection; Merckle GmbH [manufacturer], ratiopharm $\mathrm{GmbH}$ [marketing authorization holder]). Products were blinded by the external provider Hubertus Apotheke am Salzufer (Berlin), and study products were indistinguishable regarding their appearance, taste, and odour.

\section{Study Duration and Assessments}

The study duration for each patient was 11 days, comprising four site visits (V1-V4, see Table S2 in the supplementary material). During each visit, the investigators carried out a physical of 3 days (d4-d6). From day 7 (V3), patients applied the comparator product until day 10 , where the final visit (V4) took place. Treatment group 2 applied the two nasal sprays in reverse order

examination covering the general health status, ear nose throat (ENT) area (assessed by endoscopic rhinoscopy) and lungs/thorax. The status of each parameter was evaluated as 'normal' or 'abnormal' and existing abnormalities had to be described and documented by the investigators. The presence and severity of nasal edema, secretion and redness were investigated by rhinoscopy and evaluated by the rhinoscopy score ranging from $0=$ absent to $3=$ severe rhinoscopic signs. In addition, blood pressure and heart rate were measured at all visits.

The level of nasal obstruction was assessed by patients during each visit (prior to application of a study product) on a visual analogue scale (VAS) ranging from 'no obstruction' to 'worst obstruction'.

\section{Nasal Spray Sensoric Scale and Overall Assessments}

To assess sensory perceptions of the nasal sprays, patients completed paper-based questionnaires depicted as a VAS reflecting the validated nasal spray sensoric scale (NSSS) developed by Mösges et al. [7]. In addition to the given 14 items of this scale, one additional item was queried (intensity of aftertaste $15 \mathrm{~min}$ after nasal spray application). Completion of the questionnaires was done immediately (10 items), 2 min (4 items) and $15 \mathrm{~min}$ (1 item) after nasal spray administration (Fig. 2). At V1 and 
Immediately after application of nasal spray

\section{Overall impression of the nasal spray}

Not at all pleasant

Particularly

pleasant

\section{Amount of medication that runs into the throat or nose}

Very large amount

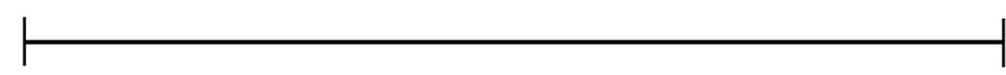

Nothing at all

\section{Extent of irritation (burning, irritation)}

Very large

\section{Need to sneeze}

Very pronounced

\section{Odour intensity}

Very strong odour

\section{Odour sensation}

\section{Extremely}

unpleasant

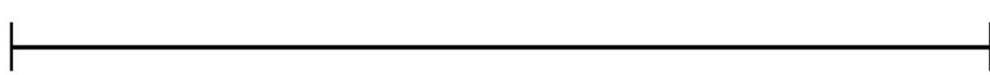

Not at all

7. Taste intensity

Very strong taste

8. Bitterness of taste

Very bitter

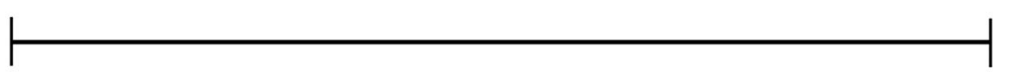

\section{Taste sensation}

Very unpleasant taste

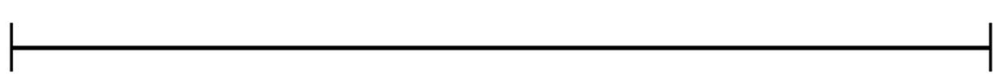

10. Nasal moisturization

Extremely dry

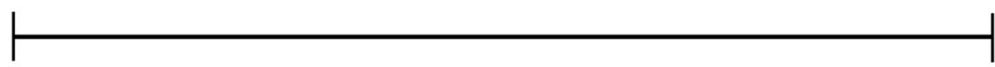

Not at all

No odour at all

Very pleasant

No taste at all

No bitterness at all

Very unpleasant taste

Extremely moist

2 minutes after application of nasal spray

\section{Intensity of aftertaste}

very strong

aftertaste

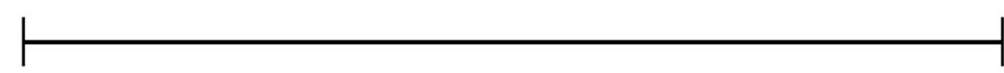

No aftertaste at all

12. Extent of irritation (burning, irritation)

Very large

\section{Amount of medication that runs into the throat or nose}

Very large amount

14. Overall impression

Not pleasant at all

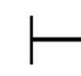

icularly pleasant

15 minutes after application of nasal spray

\section{Intensity of aftertaste}

very strong aftertaste

No aftertaste at all 
4Fig. 2 Nasal spray sensoric scale (NSSS) used during the trial adapted from [7]. The validated 14-item version of the NSSS was expanded with one additional item assessed 15 min after nasal spray application

V3, patients applied the first daily dose of the nasal spray assigned in accordance with the allocated treatment sequence at the investigational site. Patients were allowed to use their nasal spray at home before attending V2 or V4 but had to apply one spray at the site prior to completion of the questionnaire.

During the final study visit (V4), patients performed a final evaluation of treatments by judging the overall efficacy and tolerability of the two nasal sprays from 0 (not satisfactory) to 3 (very good) and by evaluating which of the two nasal sprays had been preferred (nasal spray applied during days 0-3 or during days 7-10).

\section{Study Endpoints}

The primary endpoint of the study was the difference in the total score of the sensory assessments of the NSSS (14 items) after first application of either new galenics or the comparator. The differences between the two nasal sprays were analysed independently of each other in crossover design in the assessments at $\mathrm{V} 1$ and $\mathrm{V} 3$ at the respective first application of the nasal sprays (intergroup differences). Results of the NSSS were analysed separately for the first 14 items and for all 15 items.

Therefore, intra-individual nasal sensory sum score differences within each treatment sequence were assessed and averaged. A value greater than 0 indicates an evaluation in favour of the new galenics; a value less than 0 indicates an evaluation in favour of the comparator product.

Secondary endpoints included the individual (15 items) sensory assessment of the nasal spray sensoric scale at first application.

Clinical effects were evaluated by analysis of changes in nasal obstruction, rhinoscopy scores and patients' overall assessment of efficacy and preference. Safety and clinical tolerability of nasal sprays were assessed by documentation of adverse events and by patients' overall judgement of product tolerability.

\section{Statistical Analysis and Sample Size Determination}

All statistical tests were performed bilaterally at the 5\% significance level. Frequencies and percentages of categorical variables such as mean values and standard deviations for continuous variables were reported descriptively. Analyses were performed with the SAS for Windows 9.4 software. The primary endpoint as well as other continuous parameters were analysed using a random effect model with the terms 'patient', 'treatment', 'sequence' and 'period'. The patient was considered a random term. The Student $t$ test and associated $t$ test were used to study effects of treatment sequences and groups.

For the calculation of the sample size, NSSS scores of $1200 \pm 200$ for the new galenics and of $1100 \pm 200$ for the comparator were assumed. Taking into account an alpha error of 0.05 , a power of 0.9 and a correlation coefficient of 0.5 , we calculated the total number of patients to be 44 , which was increased to 50 because of potential dropouts.

\section{RESULTS}

Overall, 51 patients were randomised, of which 26 were allocated to treatment sequence 1 (new galenics-comparator) and 25 to treatment sequence 2 (comparator-new galenics). All 26 patients of treatment sequence 1 completed the treatment. Within treatment sequence 2 , two patients only received a single application of medication during $\mathrm{V} 1$, whereas 23 patients completed the treatment (Fig. 3).

The following results reflect data of the entire intention to treat (ITT) population (randomised patients with at least complete data sets of V1 and V3, $n=49$ ) as well as data of subset populations described later. 


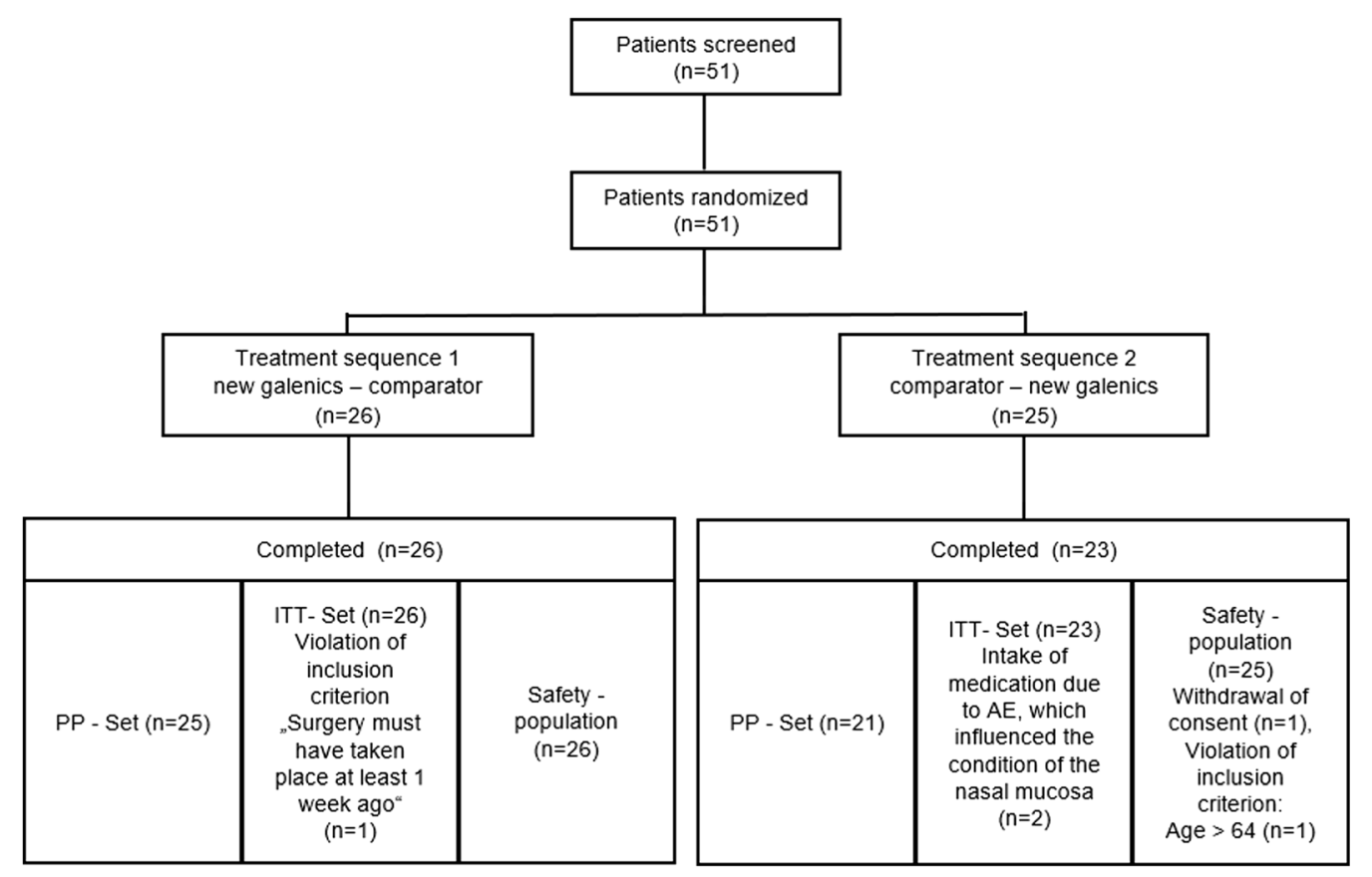

Fig. 3 Allocation of patients to analysis groups. PP per protocol population, ITT intention to treat population (including all randomised patients with a completely documented set of nasal spray sensoric scale data at V1 and V3)

\section{Demographic and Baseline Characteristics}

Demographics of participating patients are listed in Table 1. Patients aged 19 to 58 years were enrolled, and there were no statistically significant differences regarding gender, age and body mass index (BMI) between the treatment sequences.

\section{Vital Signs and Physical Examination}

At all visits, blood pressure and heart rate of patients were documented, and values were comparable between treatment sequences. All patients presented with normal general health status and normal lung/chest values at screening visit. Abnormal ENT findings (e.g. increased mucous obstruction, crusts, swelling or oedema) were observed in a subset of patients ( $n=10 ; 38.5 \%$; for treatment sequence $1, n=8$; $34.8 \%$; for treatment sequence 2 ) during the screening visit.

\section{Nasal Obstruction and Rhinoscopy}

Nasal obstruction values improved from V1 to V2 both upon treatment with new galenics (mean \pm SD, $40.31 \pm 21.24$ to $34.77 \pm 26.25$ ) and with comparator (mean $\pm \mathrm{SD}$, $36.17 \pm 24.16$ to $31.48 \pm 25.12$ ). Within treatment sequence 1 (starting treatment with new galenics), nasal obstruction values improved further, thereby reaching values of $27.38 \pm 24.23$ at V3, whereas a deterioration of nasal obstruction was observed in patients of treatment sequence 2 (starting treatment with comparator) with values of $49.09 \pm 31.07$ at V3. Thus, after treatment with the comparator product (including the washout phase) nasal obstruction worsened by a mean of 12.91 points (36.17 at V1 to 49.09 at V3). In contrast after treatment with new galenics (including washout phase) obstruction significantly improved by 12.93 (40.31 at V1 to 27.38 at V3; $p=0.008$ ) demonstrating an advantage over the comparator product (Fig. 4). Upon treatment, rhinoscopy values (sum scores including values of nasal edema, secretion and redness) improved steadily from V1 to V4 in both groups. 
Table 1 Baseline characteristics of participants

\begin{tabular}{|c|c|c|c|c|c|c|}
\hline Treatment sequence & & & $\begin{array}{l}\text { Age } \\
\text { (years) }\end{array}$ & $\begin{array}{l}\text { BMI }(\mathbf{k g} / \\
\left.\mathbf{m}^{2}\right)\end{array}$ & $\begin{array}{l}\text { Female, } \\
n(\%)\end{array}$ & $\begin{array}{l}\text { Male, } \\
n(\%)\end{array}$ \\
\hline \multirow{9}{*}{$\begin{array}{l}\text { Treatment sequence } 1 \text { (new } \\
\text { galenics-comparator) }\end{array}$} & Number & Valid & 26 & 26 & \multirow[t]{9}{*}{$9(34.60 \%)$} & \multirow{9}{*}{17 (65.40\%) } \\
\hline & & Missing & 0 & 0 & & \\
\hline & Mean & & 32.23 & 26.79 & & \\
\hline & SD & & 10.12 & 4.77 & & \\
\hline & Minimum & & 19 & 19 & & \\
\hline & Maximum & & 58 & 37 & & \\
\hline & \multirow[t]{3}{*}{ Percentile } & 25 & 25.75 & 23.08 & & \\
\hline & & 50 & 30.50 & 25.74 & & \\
\hline & & 75 & 37.50 & 30.36 & & \\
\hline \multirow{9}{*}{$\begin{array}{l}\text { Treatment sequence } 2 \text { (comparator-new } \\
\text { galenics) }\end{array}$} & \multirow[t]{2}{*}{ Number } & Valid & 23 & 23 & \multirow[t]{9}{*}{$10(43.50 \%)$} & \multirow{9}{*}{13} \\
\hline & & Missing & 0 & 0 & & \\
\hline & Mean & & 32.52 & 26.16 & & \\
\hline & SD & & 10.12 & 5.04 & & \\
\hline & Minimum & & 20 & 19 & & \\
\hline & Maximum & & 57 & 38 & & \\
\hline & \multirow[t]{3}{*}{ Percentile } & 25 & 24 & 22.44 & & \\
\hline & & 50 & 28 & 25.17 & & \\
\hline & & 75 & 41 & 30.19 & & \\
\hline
\end{tabular}

\section{Sensory Perception of the Applied Nasal Sprays}

The assessment of the sensory perception of the two nasal spray products determined by the NSSS was analysed both for the entire ITT population as well as for subgroups of patients showing abnormal findings during the ENT examination and for subgroups sorted by gender or age (below or above 30 years). Cumulative scores of the sensory evaluation of the NSSS were analysed both incorporating the first 14 items (assessment immediately and 2 min after application) and incorporating all 15 items (additional inclusion of assessment 15 min after application).
Importantly, a significant period effect was observed during the study ( $p=0.004)$ with both products being evaluated as significantly better in the second period than in the first period. The resulting limitations on the crossover evaluation of the endpoints were considered for the following study results.

Regarding the primary endpoint, the analysis of NSSS data (14 items) demonstrated overall no significant treatment effect, i.e. no differences of mean sum scores were demonstrated ( $p=0.487$, Fig. 5a).

Analysis of 15 item data confirmed the results above.

Within the subgroup of patients with nasal abnormalities (pronounced signs of inflammation; $n=10$ for treatment group $1, n=8$ for 


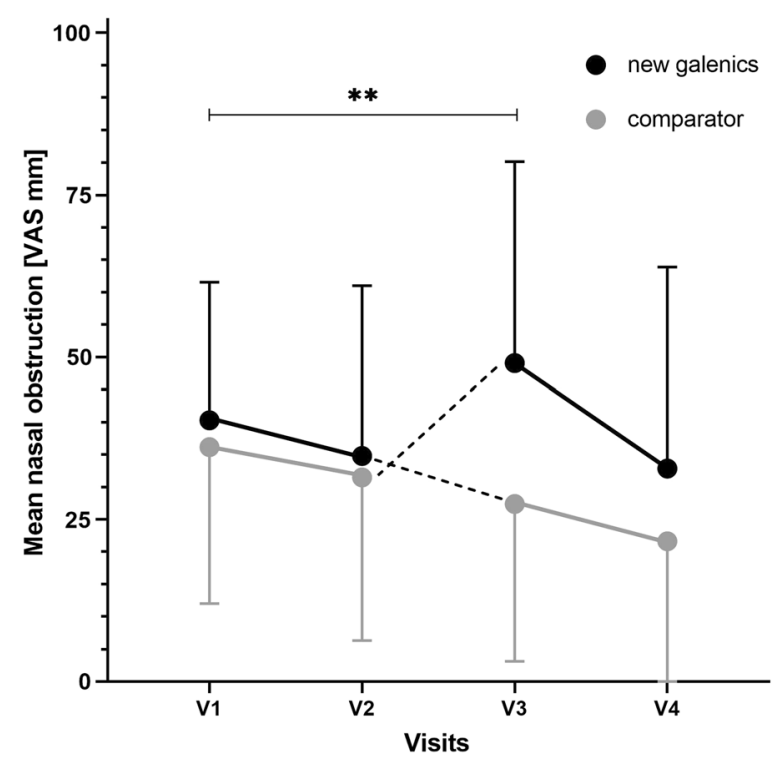

Fig. 4 Change in nasal obstruction upon treatment with new galenics or comparator. Data is expressed as mean VAS $+S D$, with ${ }^{* *} p=0.008$ for the nasal obstruction from V1 to V3 (after treatment with new galenics)

treatment group 2), no significant period effect was observed. Mean NSSS sum scores (14 items) after first application were 1149.50 upon new galenics application compared to mean NSSS

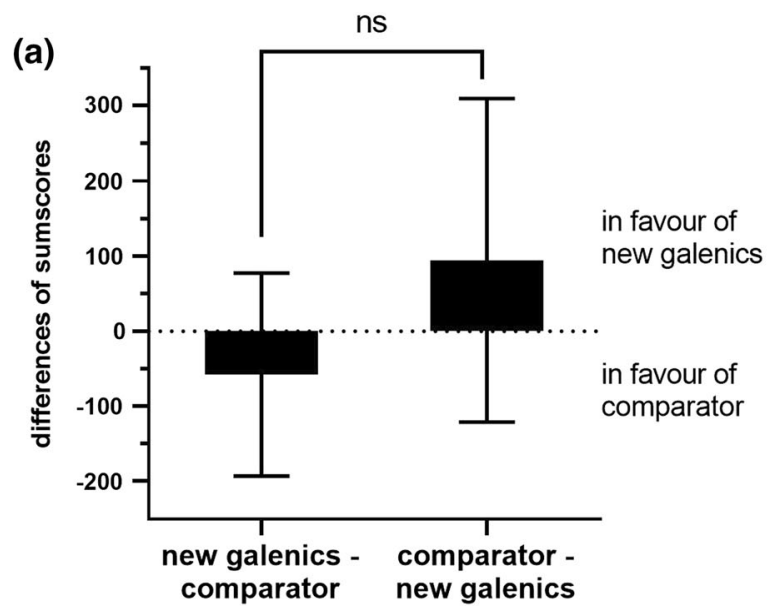

Fig. 5 Mean differences of nasal spray sensoric scale (NSSS) sum scores from V1 to V3. Patients were treated with new galenics $(\mathrm{d} 0-\mathrm{d} 3)$ followed by treatment with comparator $(\mathrm{d} 7-\mathrm{d} 10$, treatment group 1$)$ or vice versa (treatment group 2). a Analysis results of the entire ITT population $(n=49)$, b analysis of subpopulation of sum scores of 1146.60 for the comparator product in treatment sequence 1 . In treatment sequence 2 , mean NSSS sum scores (14 items) after respective first application were 1261.63 upon new galenics application compared to mean NSSS sum scores of 1120.75 for the comparator product. Overall, statistically significantly higher NSSS values were shown upon first application of new galenics compared to comparator $(p=0.033$; Fig. $5 b)$.

Subgroup analyses considering gender of patients did not show any significant treatment effects (men $p=0.733$; women $p=0.575$ considering 14 items). A significant treatment effect in favour of new galenics was shown for patients aged below 30 years $(p=0.018$ in the 14-item analysis) but not for patients aged above 30 years $(p=0.099$ in the 14 -item analysis).

The analysis of the secondary endpoints of the study investigated differences of sum scores of the NSSS over the course of the study (from $\mathrm{V} 1$ to V2 and from V3 to V4). No significant differences between treatment sequences were shown for treatment period $1 \quad(p=0.688)$ or treatment period $2(p=0.923)$.

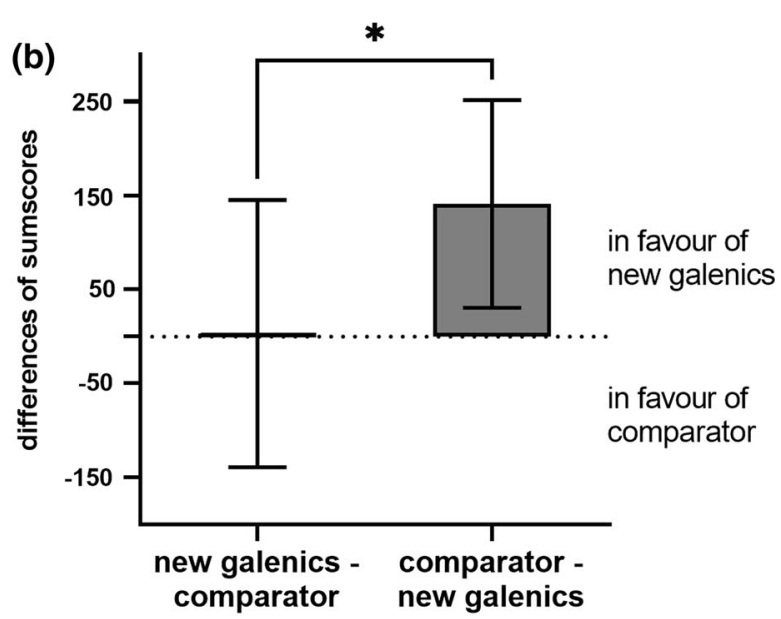

patients with nasal abnormalities $(n=18)$. Data is expressed as mean difference of sum scores + SD with ns not statistically significant and ${ }^{*} p=0.033$ 


\section{Evaluation of Single Item Scores}

The analysis of intersequence comparisons for individual sensory items revealed no significant differences between treatment sequences in any of the assessed items but a significant period effect in 8 out of the 14 items.

Analysis of single items of the NSSS revealed the greatest treatment differences of at least 4 score points for the items 2 ('amount of medication that runs into the throat or nose'), 5 ('odour intensity') and 7 ('taste intensity') in favour of the new galenics product. Differences between the new galenics and the comparator product were 6.3 for item $2,4.1$ for item 5 and 6.2 for item 7 . The sum of those three items increased significantly greater after treatment with the new galenics product compared to treatment with the comparator product $(p=0.023)$.

The item 'nasal moisturization' improved by 4.5 points ( 73.27 to 77.77 ) during the treatment with the new galenics compared to improvement of 1.85 points (77.38 to 79.23 ) during the treatment with the comparator (treatment sequence 1$)$. In treatment sequence 2 , there was an improvement of 8.96 points (71.43 to 80.39) during the treatment with the new galenics compared to a reduction by 0.79 points $(70.70$ to 69.91) during the treatment with the comparator. While no significant differences between treatments were shown for improvement of nasal moisturization in both treatment sequences (based on crossover design; Fig. 1), the improvement of nasal moisturization was significant only after treatment with new galenics during period 2 (V3-V4) $(p=0.026$, Fig. 6).

\section{Preference of Treatments}

At the end of the study, patients evaluated which of the nasal spray was preferred. In total, a significantly higher number of patients ( $n=28 ; 57.1 \% ; p=0.031)$ preferred the new galenics product over the comparator product $(n=17 ; 34.1 \%)$. The remaining 4 patients (8.2\%) had no preference (Fig. 7).

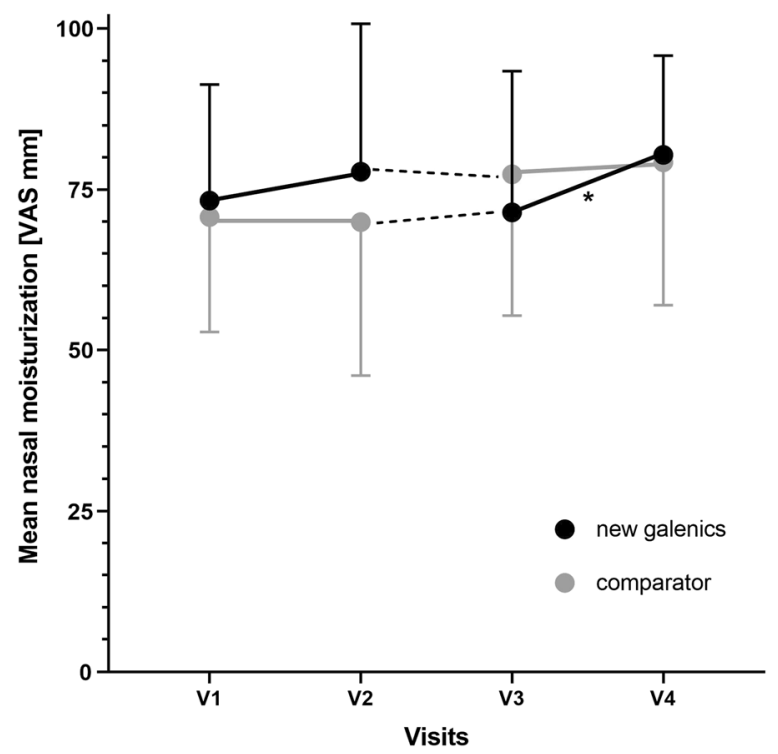

Fig. 6 Change in nasal moisturization upon treatment with new galenics or comparator. Data is expressed as mean VAS $+S D$, with ${ }^{*} p=0.026$ for nasal moisturization from V3 to V4 (during treatment with new galenics)

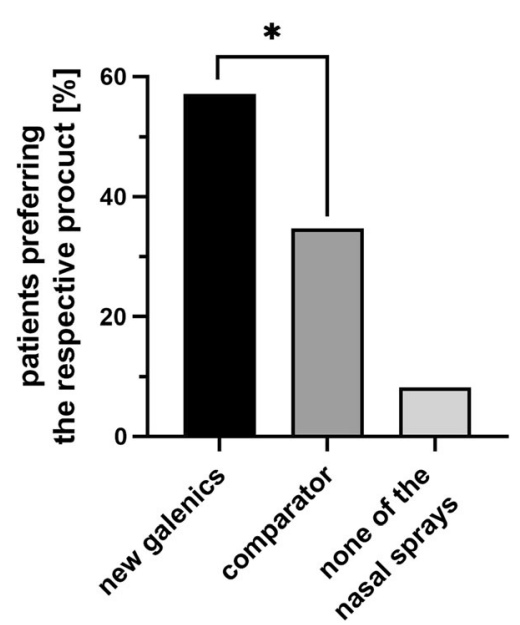

Fig. 7 Preference of products assessed by patients at V4. Data is expressed as the proportion of patients preferring either new galenics, the comparator or none of the two nasal sprays with ${ }^{*} p=0.031$

\section{Efficacy and Tolerability Assessment}

As shown in Fig. 8, the efficacy of both treatments was judged as good to very good by the majority of patients. Numerical values of percentages of patients with a good or very good 


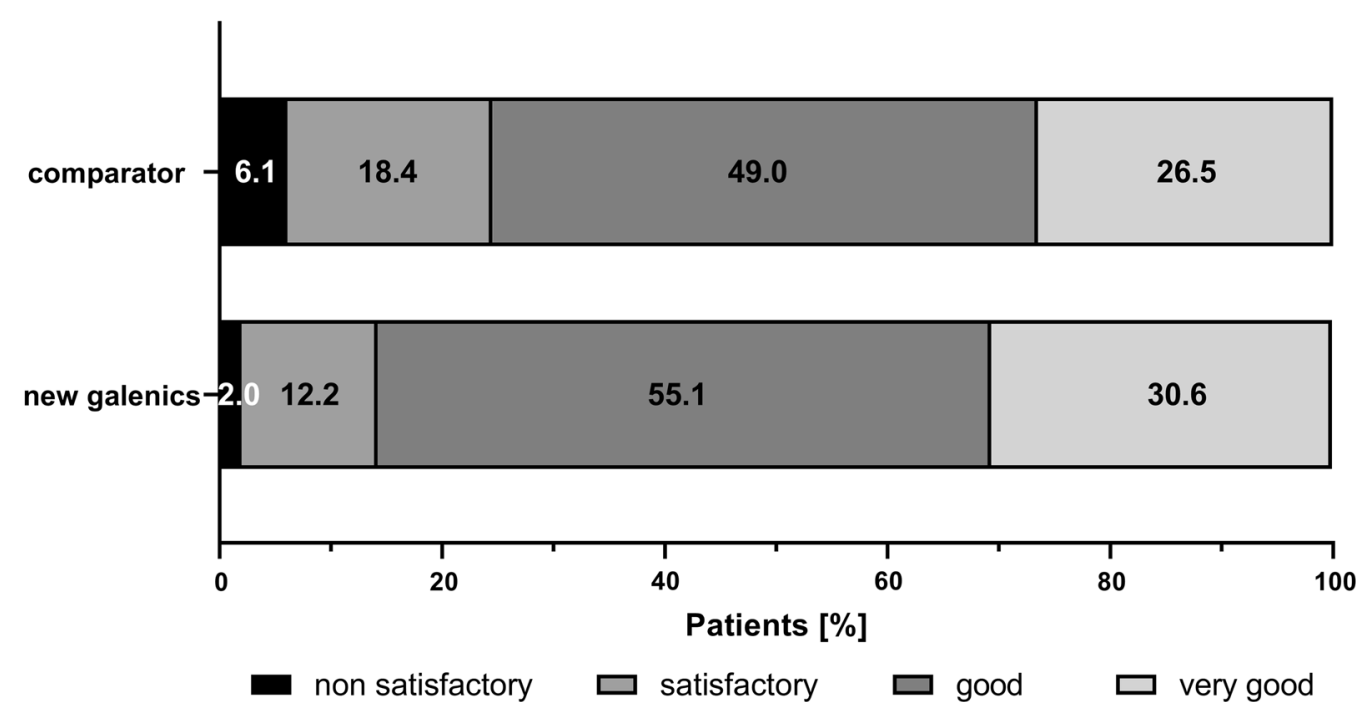

Fig. 8 Overall efficacy of study products assessed by patients at V4. Data is expressed as the proportion of patients rating the efficacy of the new galenics or comparator as either 'non-satisfactory', 'satisfactory', 'good' or 'very good'

rating of new galenics $(85.7 \%)$ were about $10 \%$ higher than those of comparator (75.5\%); however, differences were not statistically significant $(p=0.307)$.

The tolerability of both nasal sprays was evaluated as good by patients of both treatment groups without significant differences.

\section{Safety Results}

No serious adverse event occurred during the trial. A total of six adverse events (AEs) in five patients were documented (three mild, one moderate, two severe intensity), one of which (nasal burning) was rated as related to treatment with comparator, two AEs (both epistaxis) were rated as probably related to treatment (one with new galenics, one with comparator). All three AEs can be classified as expected, as they are listed in the SPCs of the products. The relationship for the other AEs was rated as unlikely or not related (Table 2).

\section{DISCUSSION}

The current study investigated the sensory perception of two decongesting nasal sprays, new galenics and comparator, in patients during convalescence from surgery on the nasal septum or nasal conchae. The use of decongestants as medical treatment after nasal surgery aims to reduce mucosal oedema by inducing vasoconstriction, and beneficial effects on nasal symptoms after septoplasty have been demonstrated [8]. However, cytotoxic and ciliary-toxic effects observed in in vitro studies may indicate a limit in decongestant application, and the risk of rebound swelling after decongestant discontinuation should always be considered [9]. The addition of dexpanthenol, an analogue of pantothenic acid with wound-healing capacities has been shown to counteract the negative effects of xylometazoline [5]. While the literature has already shown the positive contribution of dexpanthenol to xylometazolinecontaining decongestant nasal sprays [10-12], the current trial investigated the comparison of two decongestant sprays with or without the additional excipient hyaluronic acid. Hyaluronic acid is a polymer whose beneficial properties have been demonstrated in the treatment of dry nose symptoms [13] or postoperative nasal symptoms [14] with hyaluronic acid-containing medical devices.

In the current study, the sensory perception of two nasal sprays was assessed with the validated nasal spray sensoric scale (NSSS) [7], which had already been successfully applied in other studies [15]. Starting at least 1 week after 
Table 2 Adverse events (AEs) documented during the study. The description of the AEs represents the original wording (verbatim) translated into English

\begin{tabular}{|c|c|c|c|c|c|}
\hline AE description (severity) & $N(\%)$ & $\begin{array}{l}\text { Treatment } \\
\text { sequence }\end{array}$ & $\begin{array}{l}\text { Time of } \\
\mathrm{AE}\end{array}$ & $\begin{array}{l}\text { Relationship to } \\
\text { treatment }\end{array}$ & Outcome \\
\hline Headache, dizziness (mild) & $1(16.7 \%)$ & 1 & $\begin{array}{c}\text { Washout } \\
\text { phase }\end{array}$ & Not related & Recovered \\
\hline Herpes zoster (mild) & $1(16.7 \%)$ & 1 & $\mathrm{~V} 2$ & Not related & Recovered \\
\hline $\begin{array}{l}\text { Disorder of tube ventilation } \\
\text { (moderate) }\end{array}$ & $1(16.7 \%)$ & 2 & $\mathrm{~V} 2$ & Unlikely & Recovered \\
\hline \multirow[t]{2}{*}{ Epistaxis (1. severe, 2. mild) } & $2(33.3 \%)$ & 2 & V2 & Probably & Recovered \\
\hline & & & V3 & Probably & \\
\hline Nasal burning (severe) & $1(16.7 \%)$ & 2 & V1 & Definite & $\begin{array}{l}\text { Recovered, premature } \\
\text { termination }\end{array}$ \\
\hline Total & $6(100 \%)$ & & & & \\
\hline
\end{tabular}

surgery, patients were randomly assigned to 4 days treatment with one of the study products; after a 3-day washout period, patients were crossed over to 4 days treatment with the alternative study product.

The evaluation of the nasal sensory quality after the first application of the nasal spray showed that no significant differences in NSSS values could be demonstrated $(p=0.487)$. Importantly, a significant period effect $(p=0.004)$ was observed during the crossover study, thereby limiting the overall analysis of results. Overall, natural healing forces proved to have more influence on the overall sensory perception of the patients than the differences in galenics. This may be considered as one of the limitations of this study.

Interestingly, a subgroup analysis including only patients with more pronounced inflammatory symptoms demonstrated that NSSS values upon first application of new galenics were significantly better $(p=0.033)$ compared to values after first application of the comparator product. This result was independent of the sequence of product application (no period effect) in favour of the new galenics product.

A closer look at this subgroup showed that the majority of these patients was enrolled with residual abnormalities and pronounced symptoms more than 3 weeks after their nasal surgery. In contrast, the majority of "normal" patients started their study participation within the first 3 weeks after surgery. This may indicate that patients who still suffer from nasal abnormalities 3 weeks after their nasal surgery may respond particularly well to the new galenics treatment. In these patients the healing process may have been delayed and therefore a period effect does not play a role.

The analysis of a subgroup of three items of the NSSS showed that the sensory perception of the parameters 'amount of medication, that runs into the throat or the nose', 'odour intensity' and 'taste intensity' was evaluated significantly better following application of new galenics compared to application of comparator. This finding may be explained by the higher viscosity of the new product, which has been achieved by the galenics of the new formulation. The higher viscosity of the formulation may increase the retention time of the nasal spray on the nasal mucosa, thereby decreasing the amount of spray running down the nose or throat, which in turn will be perceived as less intense in taste and odour.

In line with the results above, treatments with new galenics resulted in an increased perception of nasal moisturization in both 
treatment periods with changes between V3 and V4 (treatment period 2) being statistically significant $(p=0.026)$. It is likely that the new galenics contribute to those beneficial effects on the perception of moisturization over time.

At the beginning of period 2, both treatment sequences also differed in the parameter obstruction, with a clear advantage of the treatment sequence that was pre-treated with new galenics and then received the comparator in period 2. Obstruction, measured as the patient's subjective assessment on a VAS, described the condition at the time of the examination. The assessment was made before the application of the respective nasal spray. This is another point that could have contributed to the period effect.

Literature data support these results demonstrating that the application of products with similar galenics following sinus surgery improved functional recovery and nasal oedema and crusting $[16,17]$.

The patients' final evaluation demonstrated a statistically significant preference for the new galenics product, which was perceived more pleasant by $57.1 \%(p=0.031)$ compared to the comparator product. In line with this, a greater proportion of patients $(85.7 \%)$ assessed the efficacy of the new galenics product as 'good to very good' compared to that of the comparator product $(75.5 \%)$. This finding is of particular importance as patient preference clearly plays a role in treatment compliance, as described in several studies investigating the sensory perceptions of intranasal corticosteroid sprays [16-19].

\section{CONCLUSION}

The current study demonstrated the safe application of the new galenics product in patients after nasal surgery. Importantly, the product was perceived more pleasant compared to the comparator product, which may be due to the changed galenics of the formulation. As a result, smaller amounts of medication may run down the throat or nose and may lead to decreased intensities of product odour and taste perception. Particularly patients with nasal abnormalities may benefit from the product as results demonstrated clear advantages for the new galenics treatment in this population.

\section{ACKNOWLEDGEMENTS}

Funding. The study was funded by Cassellamed $\mathrm{GmbH} \& \mathrm{Co}$. KG which also acted as sponsor. The sponsor also funded the journal's Rapid Service and Open Access Fees.

Medical Writing, Editorial and Other Assistance. Writing and editorial assistance in the preparation of this manuscript was provided by Dr. Nina Werkhäuser (ClinCompetence Cologne $\mathrm{GmbH})$. Assistance in MedDRA coding and editorial assistance was provided by PD Dr. Esther Raskopf (ClinCompetence Cologne $\mathrm{GmbH})$.

Authorship. All named authors meet the International Committee of Medical Journal Editors (ICMJ) criteria for authorship for this article, take responsibility for the integrity of the work and have given their approval for this manuscript to be published.

Author Contributions. Jennifer Lander, Lea Radtke, Laura England and Ralph Mösges designed the study. Laura England and Lea Radtke coordinated the study. Jennifer Lander participated in the conduct of the study. Christoph Bergmann conducted the study as lead investigator. Jaswinder Singh performed the data management and contributed to the data analysis. Ralph Mösges interpreted the results of the study. All authors contributed to the writing of the manuscript and approved of the final version.

Disclosures. Jennifer Lander, Lea Radtke, Laura England and Jaswinder Singh are employees of ClinCompetence Cologne who were commissioned by Cassella-med $\mathrm{GmbH} \&$ Co. KG to conduct the current clinical study. Christoph Bergmann reports personal fees and grants from Bencard Allergy, personal fees from HAL Allergy, personal fees from Sanofi 
Genzyme and non-financial support from SCS. Ralph Mösges reports personal fees from ALK, grants from ASIT biotech, personal fees from allergopharma, personal fees from Allergy Therapeutics, grants and personal fees from Bencard, grants from Leti, grants, personal fees and nonfinancial support from Lofarma, nonfinancial support from Roxall, grants and personal fees from Stallergenes, grants from Optima, personal fees from Friulchem, personal fees from Hexal, personal fees from Servier, personal fees from Klosterfrau, nonfinancial support from Atmos, personal fees from Bayer, nonfinancial support from Bionorica, personal fees from FAES, personal fees from GSK, personal fees from MSD, personal fees from Johnson \& Johnson, personal fees from Meda, personal fees and nonfinancial support from Novartis, nonfinancial support from Otonomy, personal fees from Stada, personal fees from UCB, nonfinancial support from Ferrero, grants from BitopAG, grants from Hulka, personal fees from Nuvo, grants from Ursapharm, outside the submitted work.

Compliance with Ethics Guidelines. Approval of the current study was granted by the Ethics Committee of the Ärztekammer Nordrhein (medical council North Rhine, reference number 2020061). The approval covered all participating study centres as all three study centres were located within the district of the responsible ethic committee. Therefore, in accordance with the AMG, the favourable vote of this responsible ethic committee was valid for all three study centres. The study was registered in the EU Clinical Trials Register with the EudraCT No. 2019-004936-52. The study was conducted in accordance with the principles of the current (2013) version of the Declaration of Helsinki, Good Clinical Practice (CPMP/ICH/ 135/1995), the study protocol, requirements of the German Medicines Act (AMG), the Basic Data Protection Regulation (DSGVO) and other applicable regulatory requirements. All patients provided informed consent prior to participating in this study. No data identifying single patients were included in this study; therefore, informed consent for publication is not needed.
Data Availability. The data generated during and/or analysed during the current study are available from the sponsor on reasonable request.

Open Access. This article is licensed under a Creative Commons Attribution-NonCommercial 4.0 International License, which permits any non-commercial use, sharing, adaptation, distribution and reproduction in any medium or format, as long as you give appropriate credit to the original author(s) and the source, provide a link to the Creative Commons licence, and indicate if changes were made. The images or other third party material in this article are included in the article's Creative Commons licence, unless indicated otherwise in a credit line to the material. If material is not included in the article's Creative Commons licence and your intended use is not permitted by statutory regulation or exceeds the permitted use, you will need to obtain permission directly from the copyright holder. To view a copy of this licence, visit http://creativecommons.org/licenses/by$\mathrm{nc} / 4.0 /$.

\section{REFERENCES}

1. Ciprandi G, Gelardi M. Open and clean: the healthy nose. Acta Biomed. 2019;90(2-s):4-6.

2. Matthias C. Surgery of the nasal septum and turbinates. GMS Curr Top Otorhinolaryngol Head Neck Surg. 2007;6:Doc10.

3. AWMF. Formstörungen der inneren und / oder äußeren Nase. S2k-Leitlinie 017/070: AWMF online; 2016.

4. Kehrl W, Sonnemann U. Improving wound healing after nose surgery by combined administration of xylometazoline and dexpanthenol. Laryngorhinootologie. 2000;79(3):151-4.

5. Klocker N, Verse T, Rudolph P. The protective effect of dexpanthenol in nasal sprays. First results of cytotoxic and ciliary-toxic studies in vitro. Laryngorhinootologie. 2003;82(3):177-82.

6. Greiner AN, Meltzer EO. Overview of the treatment of allergic rhinitis and nonallergic rhinopathy. Proc Am Thorac Soc. 2011;8(1):121-31. 
7. Mösges R, Pasch N, Sayar A, Schmalz P, Vent J. Survey of sensory perception and patients' subjective assessment of the application of nasal sprays the nasal-spray-sensoric-scale. Laryngorhinootologie. 2009;88(9):587-91.

8. Dağli E, Ocak E, Mirici E, Kaya M, Acar A. Effects of early postoperative nasal decongestant on symptom relief after septoplasty. Int Forum Allergy Rhinol. 2018;8(12):1476-80.

9. Fokkens WJ, Lund VJ, Hopkins C, et al. European position paper on rhinosinusitis and nasal polyps 2020. Rhinology. 2020;58(S29):1-464.

10. Mösges R, Shah-Hosseini K, Hucke HP, Joisten MJ. Dexpanthenol: an overview of its contribution to symptom relief in acute rhinitis treated with decongestant nasal sprays. Adv Ther. 2017;34(8): 1850-8.

11. Kehrl W, Sonnemann U. Dexpanthenol nasal spray as an effective therapeutic principle for treatment of rhinitis sicca anterior. Laryngorhinootologie. 1998;77(9):506-12.

12. Kehrl W, Sonnemann U, Dethlefsen U. Advance in therapy of acute rhinitis-comparison of efficacy and safety of xylometazoline in combination xylometazoline-dexpanthenol in patients with acute rhinitis. Laryngorhinootologie. 2003;82(4): 266-71.

13. Thieme U, Müller K, Bergmann C, et al. Randomised trial on performance, safety and clinical benefit of hyaluronic acid, hyaluronic acid plus dexpanthenol and isotonic saline nasal sprays in patients suffering from dry nose symptoms. Auris Nasus Larynx. 2020;47(3):425-34.

14. Gouteva I, Shah-Hosseini K, Meiser P. Clinical efficacy of a spray containing hyaluronic acid and dexpanthenol after surgery in the nasal cavity (septoplasty, simple ethmoid sinus surgery, and turbinate surgery). J Allergy (Cairo). 2014;2014: 635490 .

15. Hahn C, Böhm M, Allekotte S, Mösges R. Tolerability and effects on quality of life of liposomal nasal spray treatment compared to nasal ointment containing dexpanthenol or isotonic $\mathrm{NaCl}$ spray in patients with rhinitis sicca. Eur Arch Otorhinolaryngol. 2013;270(9):2465-72.

16. Casale M, Moffa A, Sabatino L, et al. Hyaluronic acid: perspectives in upper aero-digestive tract. A systematic review. PLoS ONE. 2015;10(6):e0130637.

17. Macchi A, Terranova P, Digilio E, Castelnuovo P. Hyaluronan plus saline nasal washes in the treatment of rhino-sinusal symptoms in patients undergoing functional endoscopic sinus surgery for rhino-sinusal remodeling. Int J Immunopathol Pharmacol. 2013;26(1):137-45.

18. Khanna P, Shah A. Assessment of sensory perceptions and patient preference for intranasal corticosteroid sprays in allergic rhinitis. Am J Rhinol. 2005;19(3):316-21.

19. Bachert C, El-Akkad T. Patient preferences and sensory comparisons of three intranasal corticosteroids for the treatment of allergic rhinitis. Ann Allergy Asthma Immunol. 2002;89(3):292-7. 